\title{
单模硫系玻璃光纤的制备及其超连续谱的产生特性
}

\author{
陈 玮 ${ }^{1,2}$, 朱 宁 $^{3}$, 赵浙明 ${ }^{3}$, 张培晴 ${ }^{2,4}$, 王训四 ${ }^{2,4}$, 戴世勋 ${ }^{2,4}$
}

(1. 邵阳学院电气工程学院, 多电源地区电网运行与控制湖南省重点实验室, 郡阳 422000; 2. 宁波大学 红外材 料与器件实验室, 宁波 $315211 ; 3$. 嘉兴学院 南湖学院, 嘉兴 314001; 4. 浙江省光电探测材料及器件重点实验室, 宁波 315211)

摘 要: 硫系玻璃具有独特的红外光学性能和极高的光学非线性等特点, 使得硫系光纤成为中红外超连续谱产生的 优选材料。基于二次挤压法制备了单模 As-S 光纤, 并利用飞秒脉冲和光参量放大器作为百浦源研究了该光纤的中 红外超连续谱的产生特性, 包括光纤长度、泵浦波长、泵浦功率对超连续谱产生的影响。结果表明该单模光纤具有 较低的传输损耗和较小的材料色散分布; 相比于传统零色散点波长附近洜浦, 在正常色散区且杂质吸收峰附近洜浦 也可获得脉冲的极大展宽(原浦参数: $4.5 \mu \mathrm{m}, 1 \mathrm{kHz}, 150 \mathrm{fs}$, 光纤长度 $23 \mathrm{~cm}$, 输出谱宽为 $1.5 \sim 8.7 \mu \mathrm{m} @ 60 \mathrm{~dB}$ 带宽); 较 短长度的硫系光纤便可产生超宽频谱输出, 相反, 光纤长度越长输出频谱越窄且平坦性变差。

关 键 词: 硫系玻璃; 单模光纤; 超连续谱

中图分类号: TN213 文献标识码: A

\section{Single-mode Chalcogenide Fiber: Fabrication and Supercontinuum Generation}

\author{
CHEN Wei ${ }^{1,2}$, ZHU Ning $^{3}$, ZHAO Zhe-Ming $^{3}$, ZHANG Pei-Qing $^{2,4}$, WANG Xun-Si ${ }^{2,4}$, DAI Shi-Xun ${ }^{2,4}$ \\ (1. College of Electrical Engineering, Hunan Provincial Key Laboratory of Grids Operation and Control on Multi-Power \\ Sources Area, Shaoyang University, Shaoyang 422000, China; 2. Laboratory of Infrared Material and Devices, Ningbo Univer- \\ sity, Ningbo 315211, China; 3. Nanhu College, Jiaxing University, Jiaxing 314001, China; 4. Key Laboratory of Optoelectronic \\ Detection Materials and Devices of Zhejiang Province, Ningbo 315211, China)
}

\begin{abstract}
Chalcogenide (ChG) glasses are well known for their unique characteristics in infrared (IR) and strong nonlinearities, endowing them as promising candidates for Mid-IR supercontinuum generation (SCG). Here, step-index As-S fiber with a small core was fabricated via twice-extrusion method. The fiber is single-mode when the propagating light wavelength is longer than $6.3 \mu \mathrm{m}$. Loss baseline of the step-index fiber is about $1 \mathrm{~dB} / \mathrm{m}$. Zero-dispersion-wavelength (ZDW) of the fiber is located at near $5.2 \mu \mathrm{m}$. By pumping the fiber with a femtosecond laser of optical parametric amplifier (OPA), Mid-IR SCG was investigated in multi-length scale fibers with different pumping wavelengths. Launching intense ultra-short pulses ( 150 fs, $1 \mathrm{kHz})$ into a 23-cm-long fiber, Mid-IR SC spanning from $1.5 \mu \mathrm{m}$ to $8.7 \mu \mathrm{m}$ can be observed when pumping at a central wavelength of either $4.5 \mu \mathrm{m}$ or $5.2 \mu \mathrm{m}$, corresponding to absorption peak at normal dispersion regime and ZDW, respectively. Besides, the spectra spanning would be extended in shorter fiber length under optimized pumping laser power. For ChG glass fiber, shorter fiber length is much more suitable for SC spanning than longer fiber length.
\end{abstract}

Key words: chalcogenide glass; single-mode fiber; supercontinuum generation

收稿日期：2017-05-18; 收到修改稿日期：2017-08-19

基金项目: 国家自然科学基金(61377099, 61627815, 61705091); 浙江省重中之重学科开放基金(xkx11508); 浙江省光电探测材 料及器件重点实验室开放基金(2017004); 嘉兴市科技局项目(2017AY13010); 嘉兴学院南湖学院科研重点资助项目 National Natural Science Foundation of China (61377099, 61627815, 61705091); Opened Key-Subject Construction Fund of Zhejiang Province, China (xkx11508); Opening Project of Key Laboratory of Optoelectronic Detection Materials and Devices of Zhejiang Province(2017004); Program for Science and Technology of Jiaxing City (2017AY13010); Key Project of Nanhu College, Jiaxing University

作者简介: 陈 玮(1980-), 男, 讲师. E-mail: 2569@hnsyu.edu.cn

通讯作者: 赵浙明, 讲师. E-mail: zhaozheming_27@sina.com 
基于光纤非线性效应产生的超连续谱具有空间 相干性好、亮度高和频谱宽等特点 ${ }^{[1]}$, 是红外波段最 具吸引力的宽带光源 ${ }^{[2]}$ 。常用的非线性光纤有石英 光纤 ${ }^{[3]}$ 、氟化物光纤 $(Z B L A N)^{[4]}$ 、碲酸盐 ${ }^{[5]}$ 和硫系光 纤 ${ }^{[1]}$ 。但是由于材料的多声子振动吸收引起的长波 限制，石英光纤的红外截止边缘小于 $2.5 \mu \mathrm{m}$, 氟化 物和碲酸盐光纤的红外截止边缘小于 $5 \mu \mathrm{m}$, 均无法 产生 $7 \mu \mathrm{m}$ 以上的超连续谱。而硫系玻璃是唯一一种 能覆盖近、中、远红外波段的玻璃材料, 且具有极高 的非线性折射率, 是石英光纤的 200 1000 倍 ${ }^{[6]}$, 因此 硫系玻璃是产生中红外超连续谱的优选材料。基于硫 系光纤的超连续谱研究已有大量实验报道 ${ }^{[7-13]}$, 但大 多以多模光纤为主, 如美国海军实验室利用光纤激 光器百浦 As-S 多模光纤获得了 $1.9 \sim 4.8 \mu \mathrm{m}$ 中红外超 连续谱输出 ${ }^{[8]}$; 丹麦科技大学利用飞秒脉冲 $+\mathrm{OPA}$ 原 浦 As-Se 多模光纤(8.5 cm 长)获得了覆盖 1.4 13.3 $\mu \mathrm{m}$ 的中红外超连续谱输出 ${ }^{[1]}$; 江苏师范大学利用飞秒 脉冲 $+\mathrm{OPA}$ 葲浦 $\mathrm{GeAsSe}$ 光纤获得了覆盖 2 10 $\mu \mathrm{m}$ 的中红外超连续谱输出 ${ }^{[9]}$; 日本丰田工业大学利用 飞秒脉冲 $+\mathrm{OPA}$ 泵浦 $\mathrm{As}-\mathrm{Se}$ 光纤 ( $3 \mathrm{~cm}$ 长) 获得了覆盖 $2 \sim 15 \mu \mathrm{m}$ 的中红外超连续谱输出 ${ }^{[10]}$; 国防科学技术 大学利用超连续谱光源泵浦 $\mathrm{As}-\mathrm{S}$ 多模光纤获得了 2 4 $\mu \mathrm{m}$ 的中红外超连续谱输出 ${ }^{[13]}$ 。近期已有单模硫 系光纤泵浦实验报道, 加拿大拉瓦尔大学通过级联 泵浦 As-Se 单模光纤获得了 3 8 $\mu \mathrm{m}$ 的中红外超连续 谱输出 ${ }^{[14]}$, 但是关于单模硫系光纤长度对超连续谱 的产生影响和参数优化尚未见报道。

本工作采用二次挤压法制备单模硫系玻璃光纤, 并采用飞秒脉冲 $+O P A$ 为泵浦源研究该硫系光纤的 中红外超连续谱的产生特性, 讨论光纤长度、永浦 波长和洜浦功率对超连续谱产生的影响。探索硫系 光纤的杂质吸收影响, 泵浦波长优化, 以及光纤长 度对频谱输出、频谱展开特性的影响。

\section{1 实验方法}

\section{1 光纤制备及性能测试}

光纤的纤芯和包层组分分别选用 $\mathrm{As}_{39} \mathrm{~S}_{61}$ 和 $\mathrm{As}_{38} \mathrm{~S}_{62}$ 玻璃。采用动态蒸馏方法 ${ }^{[15]}$ 并结合传统的熔 融一淬冷法熔制玻璃 ${ }^{[16]}$, 制备的纤芯和包层玻璃直 径分别为 $9 \mathrm{~mm}$ 和 $46 \mathrm{~mm}$, 厚度约 $20 \mathrm{~mm}$ 。采用分步 式挤压法制备光纤预制棒 ${ }^{[17]}$ : 首先通过一次挤压 ${ }^{[15]}$, 如图 1(a) (d) 获得直径约 $9 \mathrm{~mm}$ 、芯包比为 $1: 5$ 的 预制棒，然后通过第二次挤压，如图 1(e) (h) 获得芯 包比为 $1: 25$ 的光纤预制棒。最后采用真空缩棒法 ${ }^{[15]}$ 获得具有聚合物 (PES) 保护的光纤预制棒, 并置于
国产特种光纤拉丝塔进行拉丝(炉膛采用氮气保护, 拉丝温度 $340^{\circ} \mathrm{C}$ ), 制得纤芯直径约 $10 \mu \mathrm{m}$ 的小 NA 型 $\mathrm{As}-\mathrm{S}$ 光纤。

采用美国 Woollam IR-VASE 红外椭偏仪测试玻 璃的折射率; 利用 RSOFT 软件计算光纤的色散; 采 用截断法测试光纤的传输损耗, 所用仪器为配碲镉 录探测器(MCT)的美国 Nicolet 5700 型傅里叶红外光 谱仪(FT-IR, 工作范围 2.5 25 $\mu \mathrm{m}$ ); 采用日本 Keyence 超景深光学显微镜观察光纤截面; 采用美国相干 (Coherent)公司的钛宝石飞秒激光器(Mira 900)和飞 秒光学参量放大器 (OPA) 为蜃浦源测试光纤的超连 续谱产生, 光谱仪为美国 $\operatorname{FPAS}$ (探测范围: 1 16 $\mu \mathrm{m}$ ).

\section{2 超连续谱装置}

实验采用的洜浦装置与文献[11]类似，如图 2 所示, OPA 输出的激光脉冲(脉宽 $150 \mathrm{fs}$, 重复频率 $1 \mathrm{kHz}$ ), 经过 $\mathrm{CaF}_{2}$ 透镜耦合至硫系光纤, 光纤输出 端置于光谱仪入射狭缝处。整个光谱仪组成包括单 色仪、探测器和锁相放大器, 光谱仪内部配备了 4 个滤光片 (波长为 $1.9 、 3.6 、 7.0 、 11 \mu \mathrm{m}$ ) 用于消除高 阶衍射信号。为了提高信号采集速度，光谱仪采用

了 64 像素线阵碲镉永(MCT)探测器。

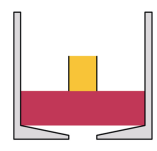

(a)

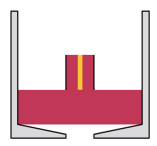

(e)

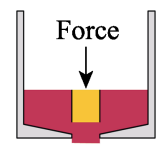

(b)

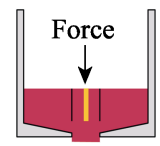

(f)

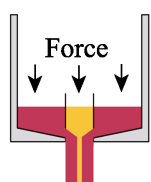

(c)

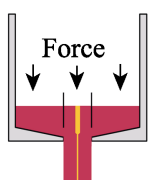

(g)

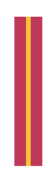

(d)
图 1 挤压流程示意图

Fig. 1 Schematic of extrusion process extruded once (a)-(c), and twice $(\mathrm{e})-(\mathrm{g})$

Yellow refers to fiber core and red refers to coating glass

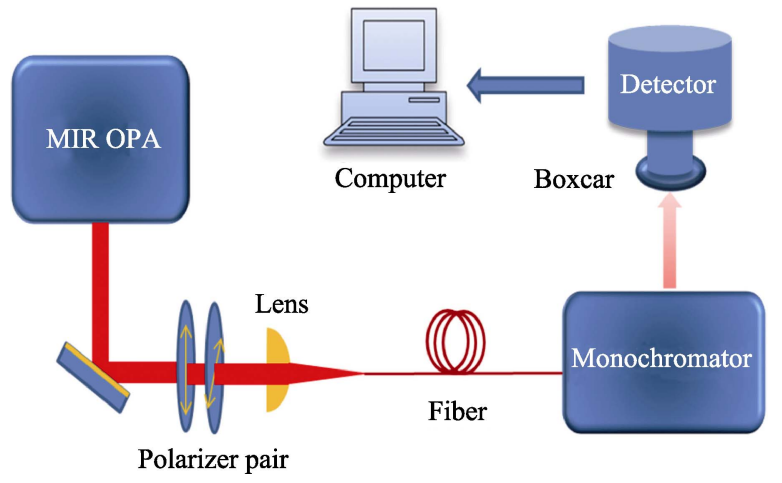

图 2 超连续谱泵浦装置示意图

Fig. 2 Experimental set-up for supercontinuum generation 


\section{2 结果与分析}

图 3(a)表示光纤的折射率分布及数值孔径 NA, 由于 $\mathrm{As}_{39} \mathrm{~S}_{61}$ 和 $\mathrm{As}_{38} \mathrm{~S}_{62}$ 的组分差异微小使得纤芯和包 层折射率差较小, 因此数值孔径分布较小, $2 \sim 10 \mu \mathrm{m}$ 波段内的 NA 始终保持在 0.5 以下; 图 3(b)表示光纤 的色散分布(黑色曲线)和归一化频率，从图中可知 光纤的零色散点波长(ZDW)位于 $5.2 \mu \mathrm{m}$ 左右; 根据 归一化频率公式

$$
V=\frac{\pi D}{\lambda} \sqrt{n_{\text {core }}^{2}-n_{\text {clad }}^{2}}
$$

其中 $D$ 为纤芯直径, $\lambda$ 为传输波长, $n_{\text {core }}$ 为纤芯折射 率, $n_{\text {cald }}$ 为包层折射率。该光纤的纤芯直径为 $10 \mu \mathrm{m}$ 。 由此可知 $\lambda \geqslant 6.3 \mu \mathrm{m}$ 时, $V \leqslant 2.405$ 即满足单模传输条 件, 如图 3(b)中蓝色点线所示。该光纤具有较小的色 散分布, 如 $3 \mu \mathrm{m}$ 处的色散绝对值约 $55 \mathrm{ps} \cdot \mathrm{nm}^{-1} \cdot \mathrm{km}^{-1}$, 而波长 $7 \mu \mathrm{m}$ 处仅为 $12 \mathrm{ps} \cdot \mathrm{nm}^{-1} \cdot \mathrm{km}^{-1}$ 。

图 4(a)为光纤的端面图, 纤芯直径约 $10 \mu \mathrm{m}$, 光 纤直径约 $250 \mu \mathrm{m}$ (除去聚合物保护层后), 从图中可 见纤芯位于截面正中心处, 未产生明显偏移, 采用 的分步式挤压法有效地避免了纤芯和包层的偏心现 象。光学损耗测试所用光纤长度为 $6 \mathrm{~m}$, 截断 $2 \mathrm{~m}$ (共截了 2 次), 该光纤的基准损耗约 $1 \mathrm{~dB} / \mathrm{m}$, 整体损 耗小于 $10 \mathrm{~dB} / \mathrm{m}$, 略高于文献[15]的实验结果。由于 FT-IR 的最小测量波长为 $2.5 \mu \mathrm{m}$, 故无法测得 $2.5 \mu \mathrm{m}$ 以下的光纤损耗谱; 光纤的工作波段可达 $6.7 \mu \mathrm{m}$, 整个损耗谱线有较小的起伏波动，除了 $4.0 、 4.25 \mu \mathrm{m}$ 处存在明显的杂质吸收峰(分别对应 S-H、C-O 杂质 吸收峰), 其余位置未见明显杂质吸收峰, 大于 $6.5 \mu \mathrm{m}$ 之后损耗急剧增大是由于多声子振动吸收引起的, 如图 4(b)所示。所有光纤的光学性能测试均采用光 纤表面涂金属镓 $(\mathrm{Ga})$ 处理, 以消除包层杂散光对性 能的影响。
图 5 为长度为 $23 、 50$ 和 $100 \mathrm{~cm}$ 的光纤所产生 的超连续谱洜浦实验结果, 为了研究零色散点波长 (ZDW) 和杂质吸收峰对超连续谱产生的影响, 研究 选取了 4.5 和 $5.2 \mu \mathrm{m}$ 两个泵浦波长。一方面, $4.5 \mu \mathrm{m}$ 位于光纤的正常色散区, 且处于 $\mathrm{S}-\mathrm{H} 、 \mathrm{C}-\mathrm{O}$ 吸收峰附 近, 而 $5.2 \mu \mathrm{m}$ 位于光纤的 ZDW 处; 另一方面, OPA 在 这两个波长的平均输出功率差异较小, 约为 $30 \mathrm{~mW}$, 脉冲宽度均为 $150 \mathrm{fS}$ 。采用相同的耦合条件进行㬌 浦实验, 若忽略不同波长输出的脉冲光斑形状变化, 可以近似认为这两个波长的泵浦功率几乎等同。

在光纤的正常色散区, 脉冲展宽首先受到自相 位调制(SPM)的作用，通过自陡效应和三阶色散的 共同作用使得光波发生分裂(OWB), 由此产生新的
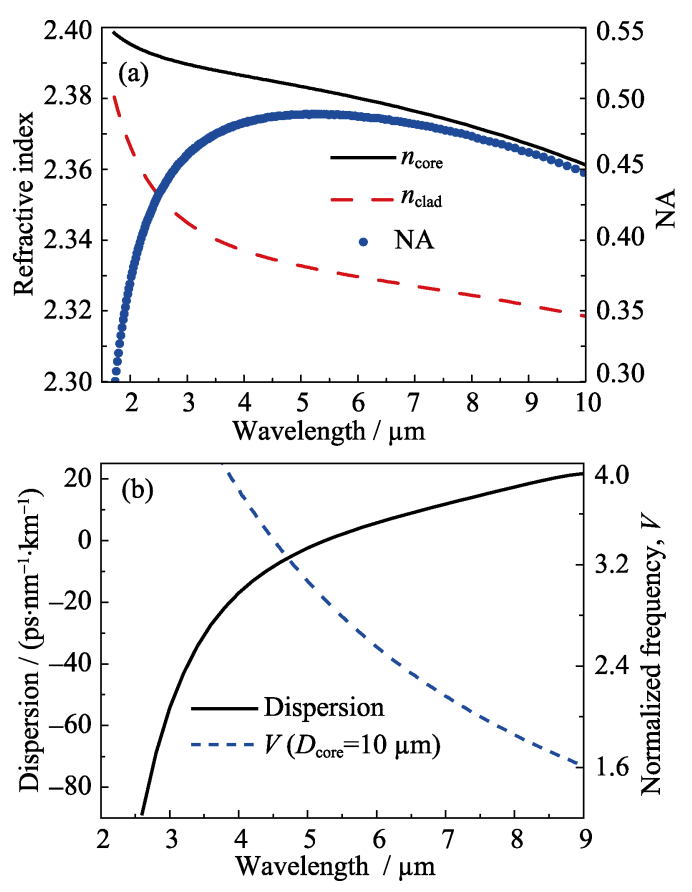

图 3 光纤参数: (a)折射率分布、数值孔径 NA; (b) 色散及归 一化频率 $V$

Fig. 3 Parameter of the fiber: (a) refractive index and NA; (b) dispersion \& $V$
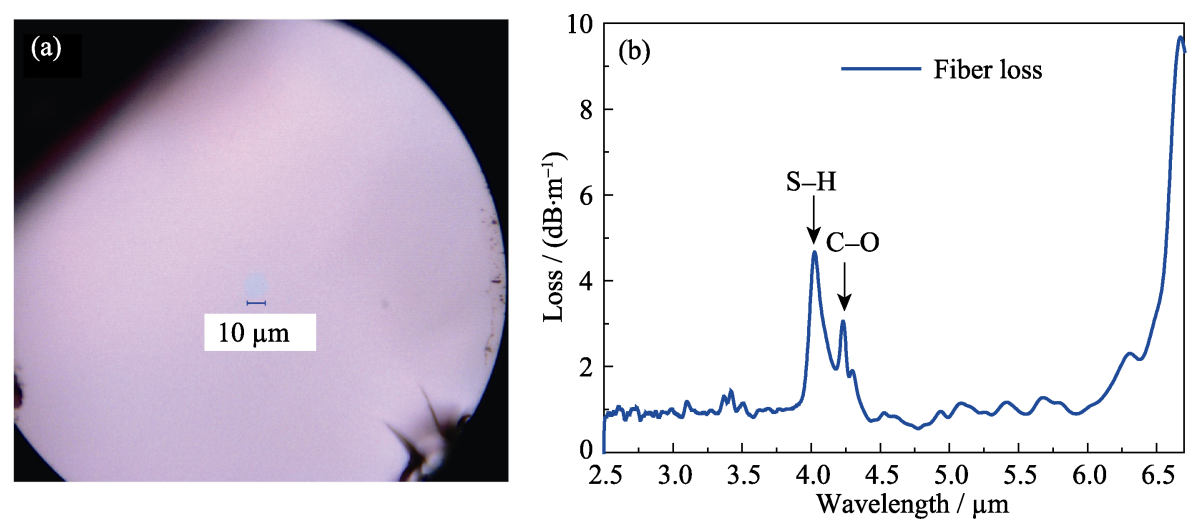

图 4 (a)光纤截面和(b)光纤损耗谱

Fig. 4 (a) Cross-section image of the fiber and (b) measured optical loss in the fiber 
频谱，从而使得频谱产生了极大的蓝移和红移，红 移部分最终越过 ZDW 进入光纤的反常色散区。在 反常色散区的脉冲展宽机制以孤子效应为主导，尤 其是拉曼效应产生的孤子自频移 $(\mathrm{SSFS})^{[3]}$ 。在光纤 的反常色散区泵浦时，与孤子有关的传输效应是光 谱展宽的主要机制：高阶孤子传输演化、孤子分裂、 孤子自频移、色散波产生和孤子俘获等 ${ }^{[3]}$ 。

图 5(a)表示在正常色散区的杂质吸收峰附近泵 浦输出的实验结果，图 5(b)表示 ZDW 附近泵浦输 出的实验结果。对比图 5(a)和(b)可知, 在光纤长度 相同的情况下, 4.5 和 $5.2 \mu \mathrm{m}$ 泵浦波长下均可实现频 谱的极大展宽(以 $60 \mathrm{~dB}$ 带宽为例): $1.5 \sim 8.7 \mu \mathrm{m}$ 和 1.5 8.7 $\mu \mathrm{m}$ (光纤长度 $23 \mathrm{~cm}$ ), 1.9 8.2 $\mu \mathrm{m}$ 和 1.9 $8.4 \mu \mathrm{m}(50 \mathrm{~cm}$ 长度), 1.9 7.2 和 1.9 7.3 $\mu \mathrm{m}(1 \mathrm{~m}$ 长 度)。考虑到这两个波长的脉冲参数(泵浦功率、脉 冲宽度)几乎相同，该结果表明：该光纤在正常色散 区的杂质吸收峰波长附近泵浦也可实现较好的脉冲 展宽效果，这与文献[18]的实验结果类似。文献[1] 获得了超宽超连续谱输出, 采用的两个泵浦波长 $4.5 \mu \mathrm{m}$ 和 $6.3 \mu \mathrm{m}$ 分别对应正常色散区和反常色散区 的杂质吸收峰。杂质吸收峰减弱了脉冲强度，从而
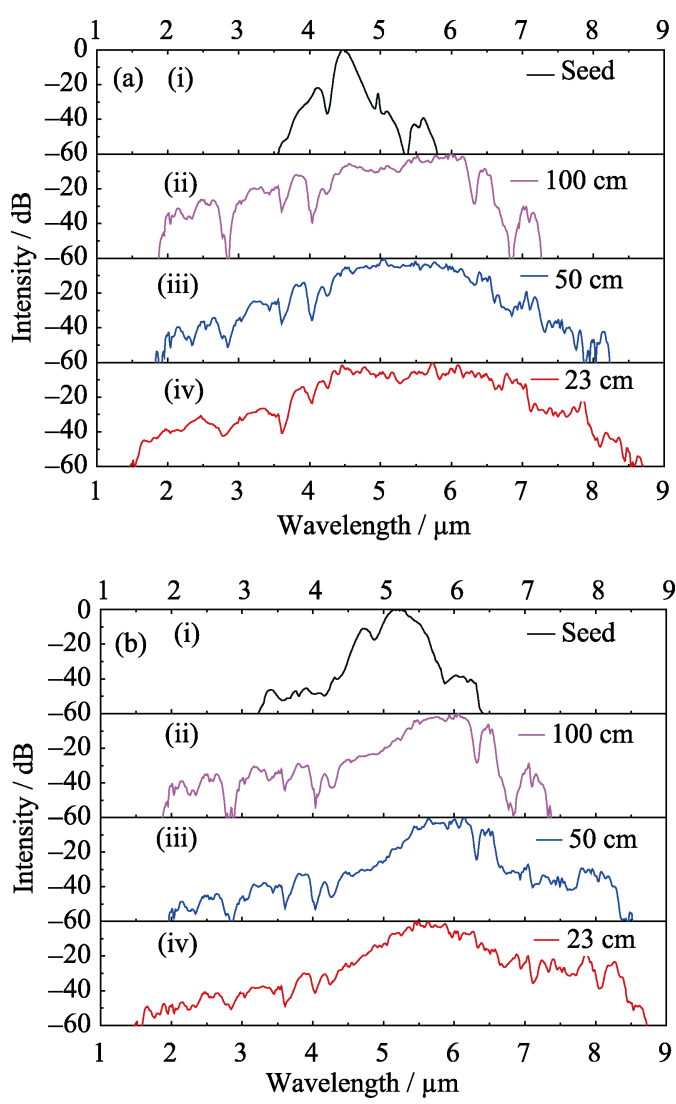

图 5 不同长度光纤的超连续谱泵浦实验结果

Fig. 5 Experimental supercontinuum generation results of fiber with different fiber-lengthes

(a) Pumped by $4.5 \mu \mathrm{m}$ at normal dispersion regime; (b) Pumped by $5.2 \mu \mathrm{m}$ at $\mathrm{ZDW}$
阻碍频谱的展宽效果 ${ }^{[8]}$ 。因此为了获得较宽的 SC 谱 输出, 硫系光纤的洜浦波长大多选择在杂质吸收峰 附近位置。

此外，得益于硫系玻璃材料的高非线性效应 ${ }^{[19]}$, 硫系光纤中的 SC 谱展宽过程主要产生在几厘米的 光纤长度内 ${ }^{[8]}$ 。因此较短长度 $(23 \mathrm{~cm})$ 的硫系光纤便 可产生极宽的频谱输出, 而光纤长度的增加反而减 小了频谱输出的宽度。这是由于硫系光纤的传输损 耗较大, 随着光纤长度的增加使得激光传输损耗增 加, 导致频谱输出展宽逐渐收窄。但相比于频谱的 蓝移部分, 红移部分受到光纤长度的影响更明显: 当波长大于 $6.5 \mu \mathrm{m}$ 后, 信号强度急剧下降, 结合光 纤的损耗分布(图 4(b)) 可得出这是由 S-S 多声子振 动吸收导致的结果。另外, 基于正常色散区的频谱 展宽应该具有较好的平坦性 ${ }^{[3]}$, 但实际的输出频谱 在 2.8、3.6、4 $\mu \mathrm{m}$ 这三个波长处存在一定的凹陷，且 随着光纤长度的增加, 凹陷更加明显, 如图 5(a)和 (b)所示。对比光纤损耗谱可知: 损耗曲线的起伏波 动(即存在的杂质吸收峰)会随着光纤长度的增加而 呈几何级数增大, 最终影响到超连续谱输出的平滑 性。进入反常色散区后，由于拉曼频移和高阶孤子 分裂使得频谱的均匀性变差, 最终表现为输出谱型 的波动起伏更大。

图 6 为不同泵浦功率下光纤的超连续谱实验结 果, 泵浦波长为 $4.5 \mu \mathrm{m}$, 所用光纤长度为 $23 \mathrm{~cm}$ 。实 验通过插入衰减片来改变泵浦激光的功率, 并用光 功率计记录。由于本实验采用了空间耦合入射方式, 无法精确地测量 OPA 激光入射至光纤的耦合效率, 因此一般采用估算值 ${ }^{[1]}$ ，为了避免耦合效率差异， 保持泵浦波长和耦合光路不变的条件下来改变功 率。如图 6 中标注为泵浦激光的平均输出功率, 分 别为 $10 、 20 、 30 \mathrm{~mW}$ ，由于只改变了入射激光的功

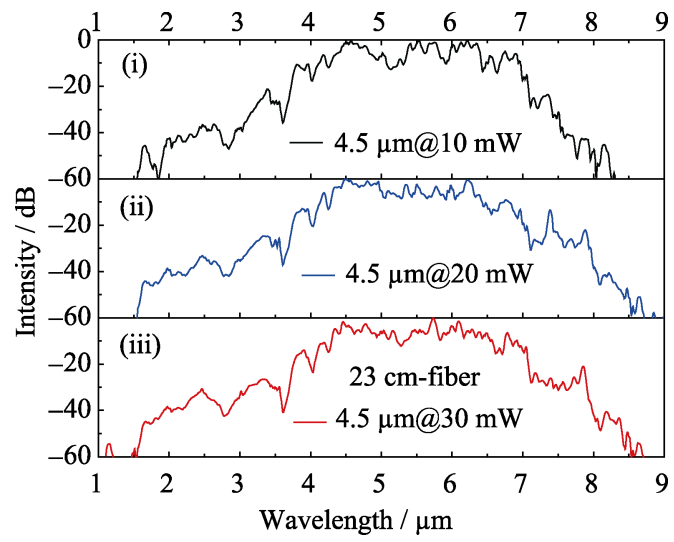

图 6 不同泵浦功率下光纤的超连续谱实验结果

Fig. 6 Experimental supercontinuum generation results of fibers under different pump-powers 
率, 脉冲波长和耦合光路未做任何改变, 因此可以 认为该情况下入射光的耦合效率也保持不变, 通过 逐步改变洜浦激光的功率, 观察输出频谱的展宽现 象。这三个泵浦功率所产生的频谱展宽 $(60 \mathrm{~dB})$ 分别 为: $1.8 \sim 8.2 \mu \mathrm{m}, 1.6 \sim 8.6 \mu \mathrm{m}, 1.5 \sim 8.7 \mu \mathrm{m}$ 。虽然洜浦功 率从 $30 \mathrm{~mW}$ 降低到了 $10 \mathrm{~mW}$, 但是频谱展宽的输出 结果差异较小, 说明当前泵浦功率已处于富余/饱和 状态(非富余/饱和状态下频谱展宽会随泵浦功率增 大而变宽)。

\section{3 结论}

利用二次挤压法成功制备了纤芯直径为 $10 \mu \mathrm{m}$ 、 芯包比为 $1: 25$ 的小 NA 型硫系玻璃光纤, 且当入 射波长大于 $6.5 \mu \mathrm{m}$ 时, 光纤满足单模传输条件。采 用飞秒脉冲洜浦源, 分别研究了光纤长度、泵浦波 长、泵浦功率对中红外超连续谱产生的影响。实验 结果表明: 较短的硫系光纤长度可以产生极宽的超 连续谱输出, 而光纤长度的增加使得声子吸收损耗 剧增, 限制了输出频谱的宽度, 同时也因为光纤传 输损耗增大, 而影响输出频谱的平坦性, 因此对于 硫系光纤而言, 短纤原浦所获得的超连续谱输出比 长纤洜浦更宽更平滑。在硫系光纤的泵浦波长选择 方面, 选择正常色散区的杂质吸收峰附近泵浦也可 获得频谱的极大展宽: 覆盖 1.5 8.7 $\mu \mathrm{m}(60 \mathrm{~dB}$ 带宽, 光纤长度 $23 \mathrm{~cm}$ ) 的超连续谱输出。该小 $N A$ 型硫系 光纤具有较低的损耗和较小的色散值, 易于泵浦获 得超宽中红外超连续谱输出。

随着未来玻璃制备工艺的完善和进步, 该硫系 光纤的损耗将进一步降低以利于实用化。因此, 基 于单模条件的硫系光纤是适用于中红外超连续谱光 源的良好非线性介质光纤。

\section{参考文献:}

[1] PETERSEN C R, MOLLER U, KUBAT I, et al. Mid-infrared supercontinuum covering the 1.4-13.3 $\mu \mathrm{m}$ molecular fingerprint region using ultra-high NA chalcogenide step-index fibre. Nat. Photonics, 2014, 8(11): 830-834.

[2] MOSELUND P M, PETERSEN C, DUPONT S, et al. Supercontinuum: broad as a lamp, bright as a laser, now in the mid-infrared.
Laser Technology for Defense and Security VIII, Maryland, USA, 2012. 83811A.

[3] DUDLEY J M, GENTY G, COEN S. Supercontinuum generation in photonic crystal fiber. Rev. Mod. Phys., 2006, 78(4): 1135-1184.

[4] JIANG X, JOLY N Y, FINGER M A, et al. Deep-ultraviolet to mid-infrared supercontinuum generated in solid-core ZBLAN photonic crystal fibre. Nat. Photonics, 2015, 9(2): 133-139.

[5] DOMACHUK P, WOLCHOVER N A, CRONIN-GOLOMB M, et al. Over $4000 \mathrm{~nm}$ bandwidth of mid-IR supercontinuum generation in sub-centimeter segments of highly nonlinear tellurite PCFs. Opt. Express, 2008, 16(10): 7161-7168.

[6] TAO G, EBENDORFF-HEIDEPRIEM H, STOLYAROV A M, et al. Infrared fibers. Adv. Opt. Photonics, 2015, 7(2): 379-458.

[7] GATTASS R R, SHAW L B, NGUYEN V Q, et al. All-fiber chalcogenide-based mid-infrared supercontinuum source. Opt. Fiber Technol., 2012, 18(5): 345-348.

[8] THEBERGE F, THIRE N, DAIGLE J F, et al. Multioctave infrared supercontinuum generation in large-core $\mathrm{As}_{2} \mathrm{~S}_{3}$ fibers. Opt. Lett., 2014, 39(22): 6474-6477.

[9] ZHANG B, GUO W, YU Y, et al. Low loss, high Na chalcogenide glass fibers for broadband mid-infrared supercontinuum generation. J. Am. Ceram. Soc., 2015, 98(5): 1389-1392.

[10] CHENG T, NAGASAKA K, TUAN T H, et al. Mid-infrared supercontinuum generation spanning 2.0 to $15.1 \mu \mathrm{m}$ in a chalcogenide step-index fiber. Opt. Lett., 2016, 41(9): 2117-2120.

[11] OU H, DAI S, ZHANG P, et al. Ultrabroad supercontinuum generated from a highly nonlinear $\mathrm{Ge}-\mathrm{Sb}-\mathrm{Se}$ fiber. Opt. Lett., 2016, 41(14): 3201-3204.

[12] ZHAO Z, WU B, WANG X, et al. Mid-infrared supercontinuum covering 2.0-16 $\mu \mathrm{m}$ in a low-loss telluride single-mode fiber. Laser Photonics Rev., 2017, 11(2): 1700005.

[13] YAO J, ZHANG B, YIN K, et al. Mid-infrared supercontinuum generation in step-index $\mathrm{As}_{2} \mathrm{~S}_{3}$ fibers pumped by a nanosecond shortwave-infrared supercontinuum pump source. Opt. Express, 2016, 24(13): 15093-15100.

[14] ROBICHAUD L R, FORTIN V, GAUTHIER J C, et al. Compact 3-8 $\mu \mathrm{m}$ supercontinuum generation in a low-loss $\mathrm{As}_{2} \mathrm{Se}_{3}$ step-index fiber. Opt. Lett., 2016, 41(20): 4605-4608.

[15] LIU SHUO, TANG JUN-ZHOU, LIU ZI-JUN, et al. Fabrication and properties of low-loss chalcogenide optical fiber based on the extrusion method. Acta Optica Sinica, 2016, 36(10): 277-284.

[16] NIE QIU-HUA, WANG GUO-XIANG, WANG XUN-SI, et al. Effect of Ga on optical properties of novel Te-based far infrared transmitting chalcogenide glasses. Acta Physica Sinica, 2010, 59(11): 7949-7955.

[17] JIANG C, WANG X S, ZHU M M, et al. Preparation of chalcogenide glass fiber using an improved extrusion method. Opt. Eng., 2016, 55(5): 056114.

[18] ZHAO Z, WANG X, DAI S, et al. 1.5-14 $\mu \mathrm{m}$ midinfrared supercontinuum generation in a low-loss Te-based chalcogenide step-index fiber. Opt. Lett., 2016, 41(22): 5222-5225.

[19] EGGLETON B J, LUTHER-DAVIES B, RICHARDSON K. Chalcogenide photonics. Nat. Photonics, 2011, 5(3): 141-148. 